\title{
A QUANTITATIVE ELECTROCARDIOGRAPHIC METHOD
}

\author{
BY \\ H. E. S. PEARSON AND L. M. GERLIS \\ From the Archway Hospital, London County Council \\ Received July 12, 1945
}

The sum of the numerous electromotive forces generated during the spread of the ventricular impulse can be regarded as a vector quantity and represented by a straight line of certain length and direction. The angle made with the horizontal by the projection of this vector on to the frontal plane is known as the electrical axis of the heart and can be calculated, by the method of Einthoven et al. (1913), from the magnitude of the initial ventricular deflections in two of the limb leads of the standard electrocardiogram.

It is possible to apply this method to an electrocardiogram derived from three points in the sagittal plane of the body, thus identifying in a similar way the sagittal projection of the same vector. From these two projections on to planes at right angles to each other, both the direction and magnitude of the vector itself can readily be calculated.

The present investigation is concerned only with the magnitude evaluated in this way, and was undertaken to explore the possibility of obtaining quantitative information on the state of the ventricular muscle. The values obtained are empirical, as only a small and unknown proportion of the total electromotive force generated by the heart is induced at the periphery and becomes available for measurement. It is not, however, unreasonable to assume that, if obtained under standard conditions, these values will bear a fixed relationship in the same patient to the resultant potential difference generated during ventricular systole.

\section{The SagitTal Electrocardiogram}

The derivations of this cardiogram must be so chosen that the projection obtained from it of the vector on to the sagittal plane is concordant with the frontal projection given by the limb tracings. The triangles formed by vertebra prominens, xiphisternum, and sacrum, and by the xiphisternum, twelfth thoracic vertebra, and forehead, were tried and found unsatisfactory in this respect, probably because the xiphisternal point is too close to the heart. A sagittal tracing derived from the triangle formed by the manubrium sterni, fourth thoracic spine, and left leg, with the terminals R.A., L.A., and L.L. connected in this order was found to be suitable and was generally adopted. In this triangle the horizontal lead lies in the same plane as lead I of the standard tracing, and it is also convenient in operation, as the five electrodes for the two cardiograms can be applied at the same time and the change-over effected by a double-pole double-throw switch. As the vector is usually directed downwards and backwards, this arrangement of the terminals gives positive deflections in each of the leads, which will be referred to as A, B, and C respectively. Standard electrodes and Cambridge jelly were used for all the skin contacts.

Calculation. The symbols I, II, III, and A, B, and C, will be used to denote the positive height, in millimetres, of the initial ventricular deflections in the respective leads, or the algebraic sum where the deflection is diphasic.

Fig. 1 shows an imaginary rectangular prism, with faces lying in the frontal, sagittal, and horizontal planes, constructed about the diagonal AG, which represents, in direction and length, the apparent electrical vector of the heart. AF and AC show the sagittal and frontal projections of this vector. Now the length AG is equal to the square root of the sum of the squares on the three dimensions of the prism. The horizontal dimensions AE, AD, have 


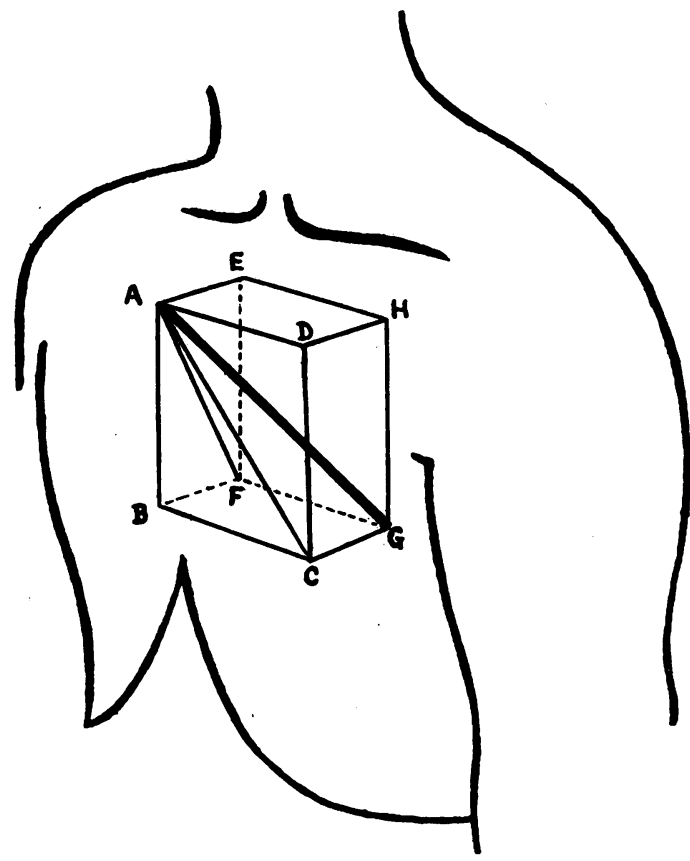

Fig. 1.-The electrical vector and its projections on to the frontal and sagittal planes.

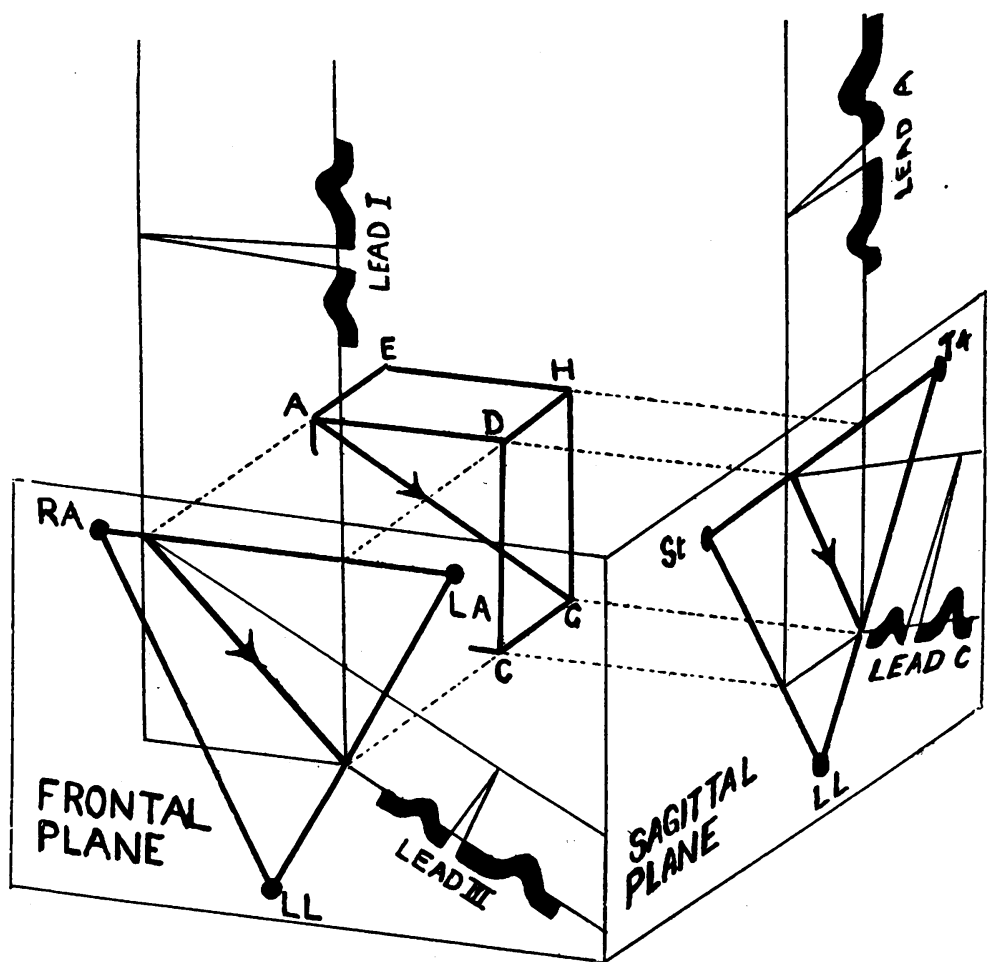

FIG. 2:- -Illustrating the construction on which the calculation of $\mathrm{V}$ is based. The triangles are equilateral and the lettering $A-G$ corresponds to that in Fig. 1. 
the numerical values $\mathrm{A}$ and I respectively, and the vertical $\mathrm{AB}$ can be calculated from either cardiogram (see Fig. 2) as $\frac{I+2 I I I}{\sqrt{3}}$ or $\frac{A+2 C}{\sqrt{3}}$. Hence the length of the vector $(V)$ is:-

$$
\sqrt{\mathrm{I}^{2}+\mathrm{A}^{2}+\frac{(\mathrm{I}+2 \mathrm{III})^{2}}{3}} \text { or } \sqrt{\mathrm{I}^{2}+\mathrm{A}^{2}+\frac{(\mathrm{A}+2 \mathrm{C})^{2}}{3}} \text {. }
$$

These alternative formulæ can be used to check the accuracy of the recordings in a given case. Exact agreement was not expected for several reasons, both theoretical, as the method attempts to simplify very complex phenomena, and practical, such as inaccuracy of standardization, artefacts of various kinds, and respiratory variations in amplitude.

In the 188 pairs of recordings considered here, substitution into the second of these two formulæ gave a higher value for $\mathrm{V}$ than was obtained from the first in 56 per cent, equality to the nearest $\mathrm{mm}$. in 30 per cent, and in 14 per cent the first formula gave the higher result. If the difference exceeded one-fifth of the higher figure, the maximum that could be attributed to the summation of instrumental errors, the result was discarded. This occurred in 16 (8.5 per cent) of the cases. Otherwise a mean value for $\mathrm{V}$ was taken if any difference existed.

A second check can be made by recording all six leads of the cardiograms instead of only three (I, III, and A or I, A, and C) required for the calculation; inaccuracies of standardization can then be detected by substitution into the formulæ $\mathrm{I}+\mathrm{III}=\mathrm{II}$ and $\mathrm{A}+\mathrm{C}=\mathrm{B}$.

The usual standard fibre sensitivity of one centimetre deflection to the millivolt was used, but for convenience the values $\mathrm{V}$ are expressed in millimetres.

\section{FindingS in Normal SubJects}

Sagittal and limb tracings were recorded in a series of 50 healthy men and women between 18 and 70 years of age, and the value of $\mathrm{V}$ calculated in each. Lying between extremes of 6 and $26 \mathrm{~mm}$., these showed a peak incidence at 11-15 mm., thereafter falling off towards the higher values (see Fig. 3). A figure below $10 \mathrm{~mm}$. was found in only two of these normal subjects; in one of them it was $9 \mathrm{~mm}$., and in the second $(6 \mathrm{~mm}$.) the tracings showed an unusual mode of spread of the contractile stimulus through the ventricles. It was not found possible to establish any relationship between an individual's $V$ value and any other physical characteristic such as age, height, weight, height/weight ratio, body surface area, or the size of the heart as estimated by the frontal X-ray area.

Tracings, which were discarded as unreliable by the standards indicated above, were obtained from a further 6 normal subjects. Most of these showed evidence of faulty recording, but their $\mathrm{V}$ values, approximately estimated, were found to fall in the same groups of maximal incidence as the rest, and their inclusion would not alter the balance of the chart.

The value of $\mathrm{V}$ was not found to change in the same normal subject at different times or at varying pulse rates.

\section{Findings in AbNormal SubJects}

The double electrocardiograms were recorded in a series of 101 consecutive patients who were known to have some cardiac disorder and the value of $\mathrm{V}$ calculated in each. These cases were as follows :

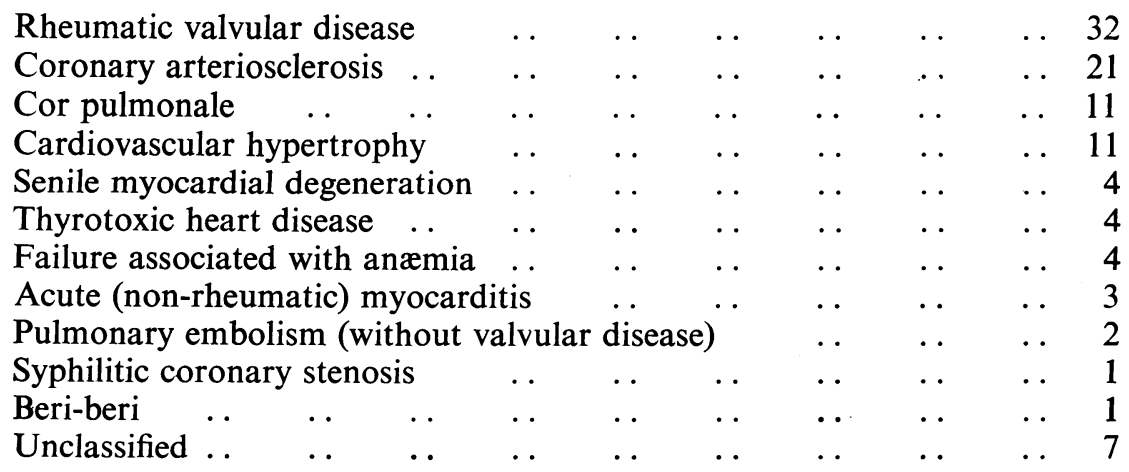


Ninety-one of these patients were further classified into four grades according to the severity of their symptoms.

0 . Symptom-free patients, in whom heart disease was discovered during routine examination.

I. Mild symptoms; e.g. reduced exercise tolerance, or first hæmoptysis in mitral disease.

II. Moderate symptoms; e.g. early congestive failure, or repeated anginal attacks.

III. Severe symptoms; e.g. bedridden patients with advanced failure.

These results, with an analysis of their statistical significance, are given in the table and frequency distribution charts below, where it will be seen that the abnormal cases as a whole show much lower values than are found in health. Taking $10 \mathrm{~mm}$. as a critical point, $45 \mathrm{per}$ cent of the patients give figures below this level, as against 6 per cent of the normal subjects. It is also apparent that the tendency to provide lower $\mathrm{V}$ values increases with the severity of the symptoms, and that those patients who are symptomless or only mildly incapacitated show no significant variation from the normal mean. Cases of rheumatic valvular disease and coronary ischæmia have been tabulated separately to show how this tendency is increased in these groups.

TABLE I

Analysis of Findings in 151 Subjects

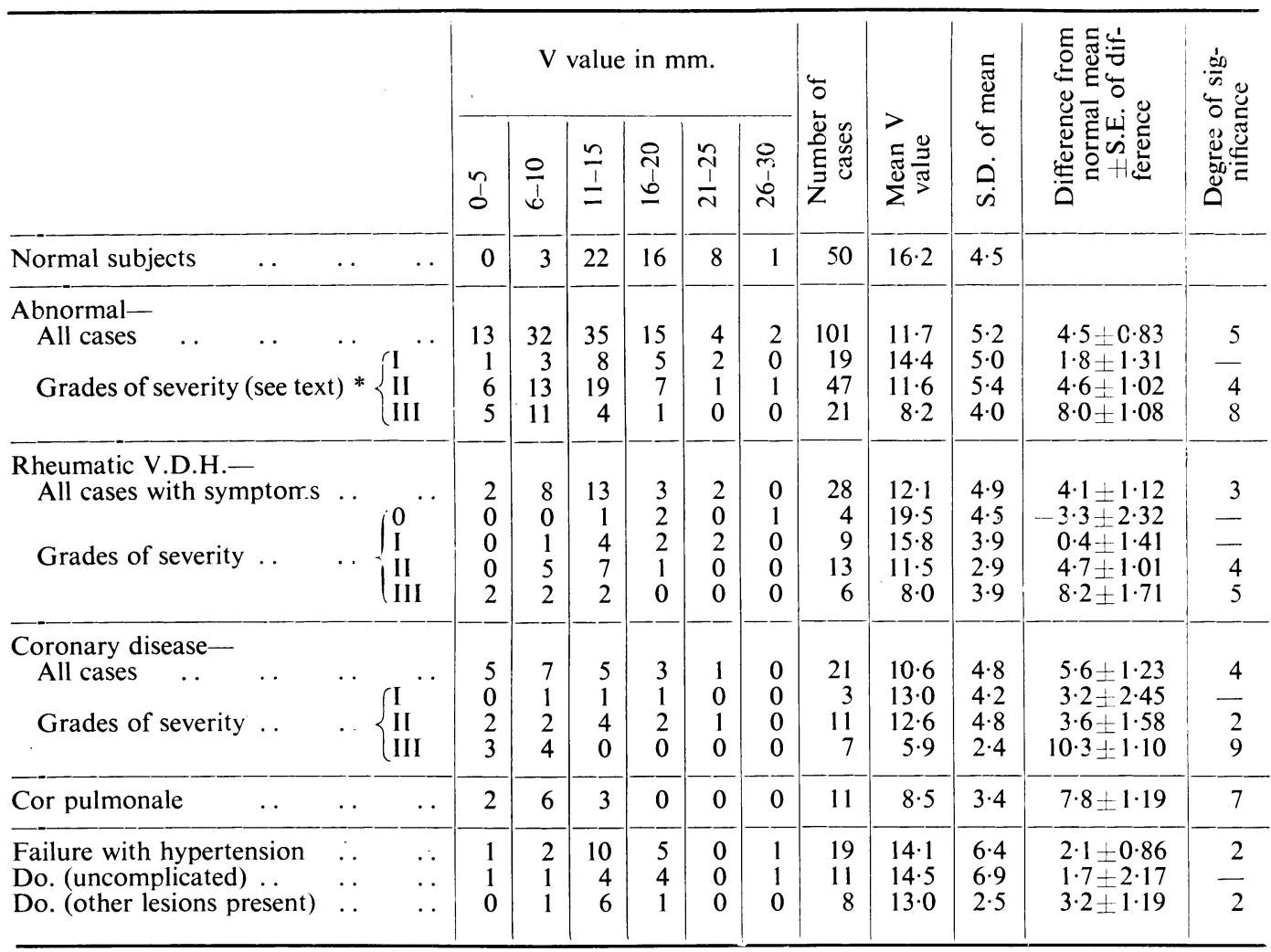

* 91 cases were graded : 4 in grade 0 are not included here.

This measure of agreement between the depression of $\mathrm{V}$ and the severity of the symptoms was not found in hypertensive patients except where other complications such as coronary stenosis, cor pulmonale, or valvular disease were present. This may be because of the greatly increased ventricular bulk in such cases, or because of the relative inadequacy of even a powerfully acting heart against the increased peripheral resistance. It is important too to note that 8 of the 19 tracings from hypertensive patients showed the broad initial ventricular deflections of the bundle branch block type. This kind of tracing presents a special source of inaccuracy, because the electromotive force is really proportional to the 

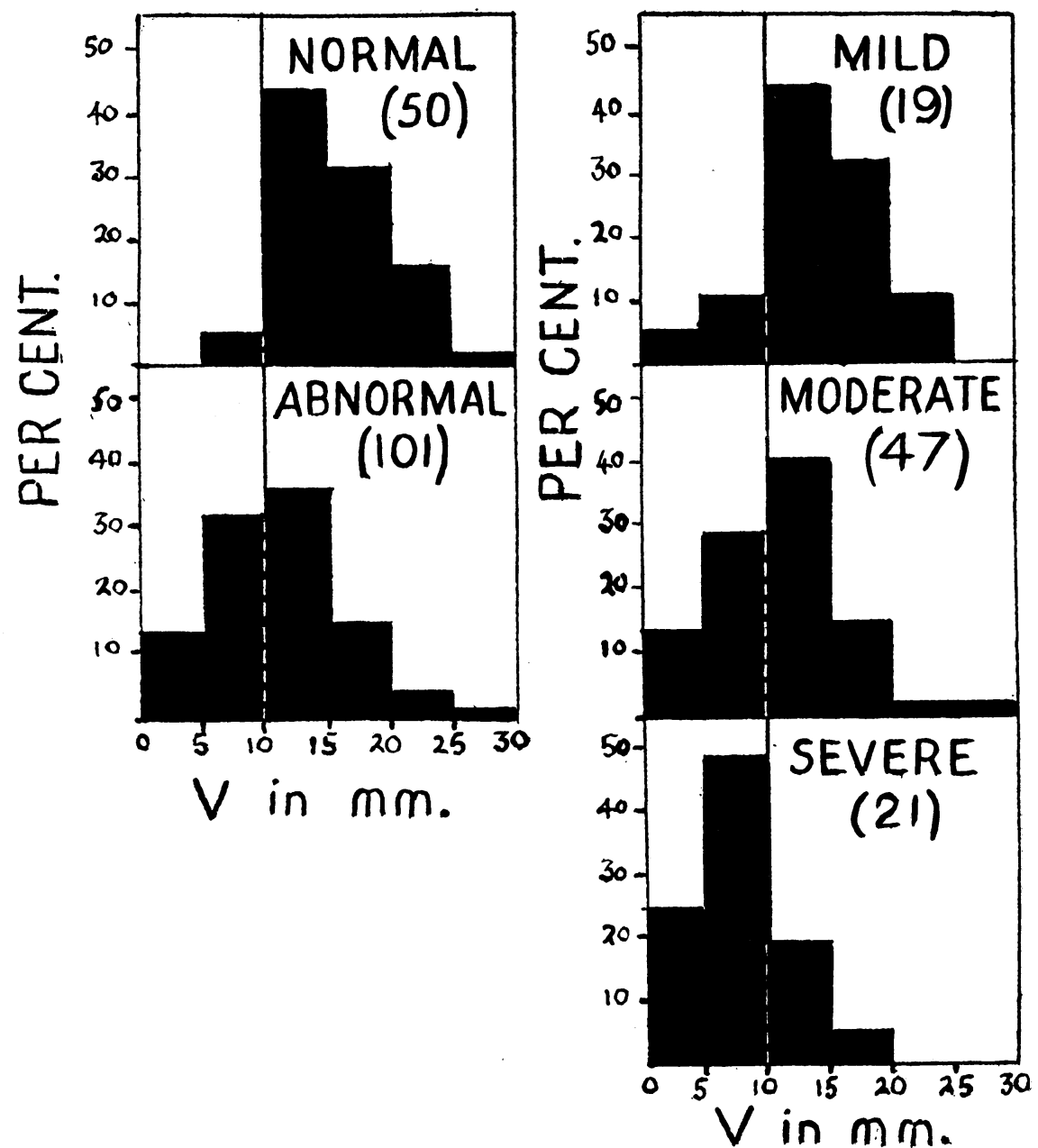

Fig. 3.-Frequency distribution charts to show the contrast between $(a)$ normal and abnormal subjects, and (b) abnormal subjects graded by severity of symptoms. Bracketed figures show the number in each group.

area contained by the initial deflection, and the use of the linear height measurement is a convenient approximation only reliable with waves of normal duration.

Repeated evaluations of $\mathrm{V}$ were made at later dates in 17 of the patients in whom there was evidence that the cardiac capacity had improved or deteriorated in the interim. Of 15 patients with clinical improvement 12 showed a higher value on the later occasion, with increases up to 60 per cent of the original figure in two cases. A difference of less than 10 per cent was disregarded. These 12 cases were of diverse pathology, and three examples will be given.

1. Male, aged 60. 25/1/45. Chronic bronchitis, early cor pulmonale, B.P. 128/80. $\mathrm{V}=10 \mathrm{~mm}$.

$15 / 2 / 45$. Much improved by rest, B.P. $155 / 80$. $V=15 \mathrm{~mm}$. (+ 50 per cent).

2. Male, aged 64. 18/1/45. Pernicious anæmia (hæmoglobin 49 per cent), with congestive failure. $\mathrm{V}=5 \mathrm{~mm}$.

30/5/45. Hæmoglobin 90 per cent, no evidence of failure. $V=8 \mathrm{~mm} .(+60$ per cent).

3. Male, aged 8. 23/5/45. Pharyngitis and cervical adenitis with toxic myocarditis, pyrexia. Cardiogram showed $P-R$ interval of 0.24 sec., occasional sinus arrest. $V=13 \mathrm{~mm}$.

31/5/45. Temperature settling. $\quad P-R, 0 \cdot 16 \mathrm{sec}$. $\quad \mathrm{V}=17 \mathrm{~mm}$.

7/6/45. Discharged from hospital. Normal rhythm, P-R, $0 \cdot 12 \mathrm{sec} . \mathrm{V}=19 \mathrm{~mm}$. ( +45 per cent $)$.

The last case is of interest because of the parallel recovery of the A-V block, a guide not always available in acute myocarditis. 


\section{RHEUMATIC} VALVULAR DISEASE CORONARY DISEASE
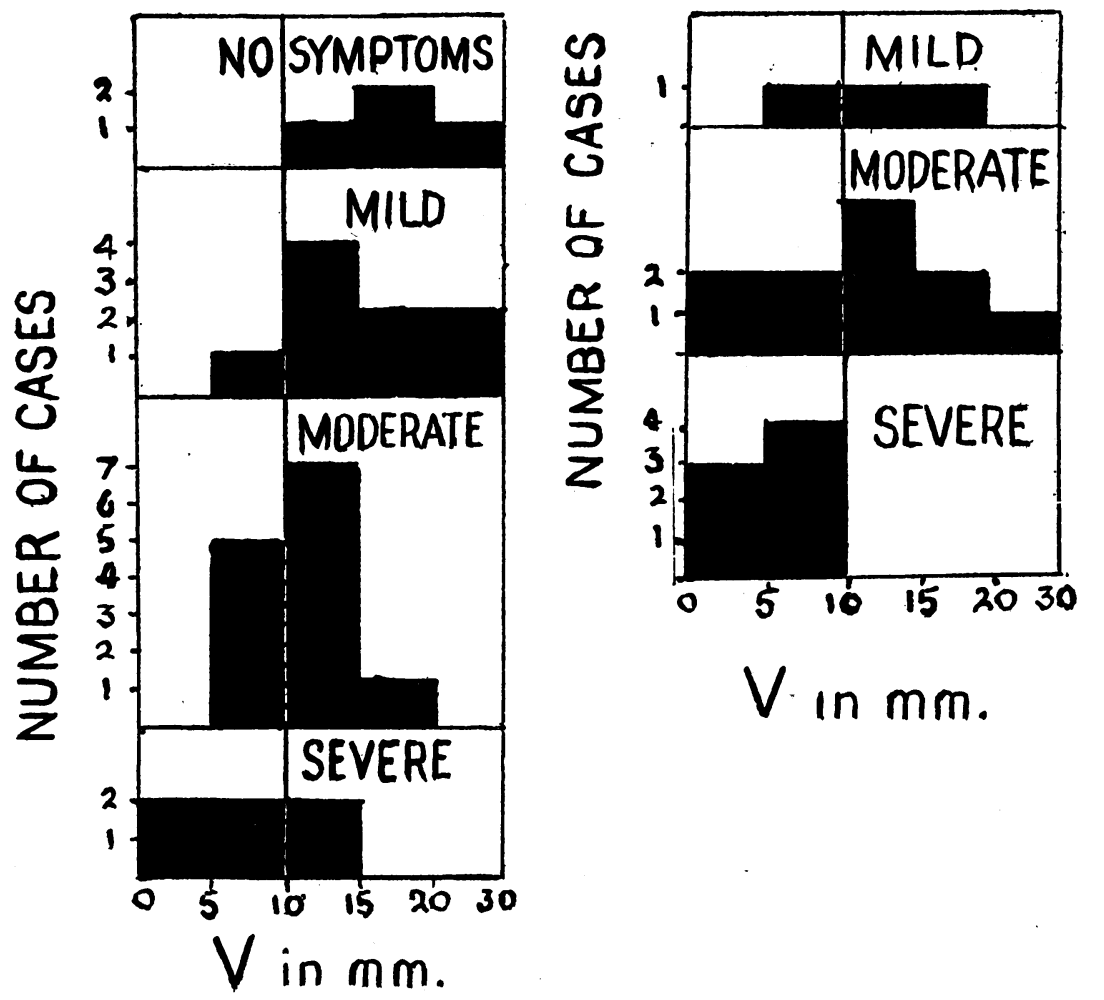

FIG. 4.-Frequency distribution in cases of rheumatic and coronary disease, graded by severity of symptoms.

Of the three cases in which the $\mathrm{V}$ value remained unaltered during apparent clinical improvement, one was an arteriosclerotic man of 70 with a hypochromic anæmia and bundle branch block; the second, a man of 33, where, after a coronary thrombosis one year previously, the improvement followed ambulant treatment of his odema by diuretics; and the third, a man with Addison's disease, whose blood pressure had risen from 110/60 to 150/90 after a desoxy-corticosterone acetate implant. These patients had little in common, but it is possible that improvement in cardiac function was not the chief cause of their better health.

The two cases, both with coronary occlusion, who had deteriorated, showed falls in $\mathrm{V}$ of 20 per cent and 75 per cent respectively. The first presented no definite clinical change, with a variable blood pressure, but the cardiographic pattern showed regression and another occlusive attack followed three days after the second tracing.

\section{Discussion}

The principal value of the clinical electrocardiogram is to provide information on abnormalities of rhythm and on disturbances of conduction of the contractile impulse. It is with the object of supplementing this by a quantitative figure based on the total resultant electromotive force generated during ventricular systole that the above method is put forward. It is realized, in the first place, that this resultant force itself, the summation of an electrical system of vast complexity, bears no well-defined relationship to the capacity of the heart to do work, and further that one measures only that proportion of the total that can be derived from peripheral electrodes. The method is therefore an arbitrary one, to be judged from a practical standpoint.

Although there is wide personal variation in the normal heart, it appears to be exceptional 
for the $\mathrm{V}$ value to fall below $10 \mathrm{~mm}$. or to exceed $25 \mathrm{~mm}$. in health. There is a much wider scatter in cases of heart disease, but it is clear that low values are in general associated with increased degrees of ventricular disease or embarrassment rather than the opposite.

If, therefore, $\mathrm{V}$ is found to alter during a patient's illness, the direction and extent of this change might be used to assess the severity of the disease as it affects the ventricular muscle, as a measure of progress and as a guide to prognosis. Further, a rising figure during recovery from an illness may permit a retrospective diagnosis of transient myocardial damage to be made where the standard tracing has shown no abnormality. The magnitude of the variations of $\mathrm{V}$ observed in changing clinical states encourages the belief that myocardial involvement may be recognized in this way in cases where failure is absent and where other clinical methods give no information.

Although the direction of the vector is not discussed in this paper, it may be mentioned again here that the combination of sagittal and frontal electrocardiograms enables the observer to fix in space the vector itself instead of simply a frontal projection of it. In this way "axis deviation" in the sagittal plane can be studied. This is of value in cases of ventricular preponderance, as anatomically the main bulks of the respective ventricles lie more in a fore-and-aft plane than transversely, and the sagittal axis is less. likely to be disturbed by variations in build and diaphragmatic level.

Another incidental finding in this work was the occasional elucidation of tracings that showed very small deflections in the limb leads. These low-voltage curves were twice found to be due to the rotation of the vector away from the frontal plane, so that it lay almost at right angles to it, giving in the limb leads the equivalent of a foreshortened image. One such subject showed in all the limb leads the prolonged, low, polyphasic initial deflections often attributed to arborization block. His sagittal cardiogram showed broad, high, monophasic deflections whose measurements indicated that the electrical vector was of normal dimensions but pointed directly antero-posteriorly. This suggests an explanation of the high-voltage præcordial tracings often recorded in such cases.

\section{SUMMARY}

A method of recording a sagittal electrocardiogram is described.

From the measurements of the initial ventricular deflections in this and the standard frontal electrocardiogram the magnitude and direction can be calculated of the vector quantity that represents the total electromotive force generated during ventricular systole.

The apparent magnitude of this vector in 50 normal subjects and 101 patients with heart disease -is given, and the significance of the findings in various grades of cardiac disability is discussed.

The value is shown generally to rise or fall in accordance with clinical estimations of the functional capacity of the heart, where this undergoes change in the same patient.

Possible clinical applications of the method are suggested.

We wish to thank the many friends who have given their time to act as volunteers, the staff of St. Mary, Islington, Hospital (L.C.C.), where most of the work was done, for their kind co-operation, and especially Sister $\mathrm{H}$. Pound for her valuable technical assistance in making the recordings.

\section{REFERENCE}

Einthoven, W., Fahr, G., and de Waart, A. (1913). Pflüger's Arch. ges. Physiol., 150, 275. 